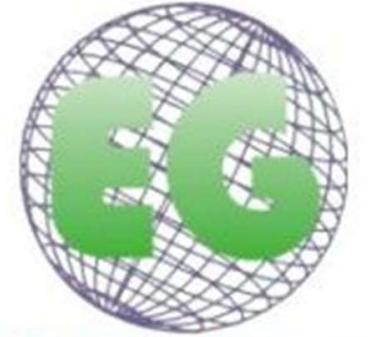

ISSN 1695-6141 N58

\title{
Estudio de las diferencias culturales en las relaciones violentas de pareja de España y Perú
}

Study of cultural differences in violent intimate partner relationships in Spain and Peru

Juan Francisco Rubio Laborda ${ }^{1}$

Pilar Almansa Martínez ${ }^{1,2}$

José Vicente Navarro Henarejos ${ }^{3}$

María del Mar Pastor Bravo ${ }^{1,2}$

${ }^{1}$ Departamento de Enfermería. Universidad de Murcia.España.

2 IMIB- Hospital General Universitario Virgen de la Arrixaca. Murcia, España.

${ }^{3}$ Hospital General Universitario Santa Lucía. Cartagena, Murcia, España.

\section{https://doi.org/10.6018/eglobal.364641}

Recibido: 28/02/2019

Aceptado: 29/04/2019

\section{RESUMEN:}

Objetivo: Determinar la violencia de género sufrida y ejercida en función de la cultura, edad y nivel educativo.

Metodología: Estudio transversal descriptivo con una muestra de 130 sujetos seleccionados aleatoriamente mediante el muestreo no probabilístico por cuotas en los países de España y Perú, siendo 53 hombres y 77 mujeres, sobre los que se aplicó un test de valoración de relaciones violentas de pareja, llamado el "semáforo de la violencia".

Resultados: Se encontraron asociaciones significativas según países en los ítems relacionados con el control de la vestimenta y de las salidas, el castigo mediante indiferencia, los celos, la sobreprotección y el control a través del móvil. Por otra parte, la educación formal genera una disminución de la violencia de género sufrida en mujeres. Respecto a la edad, los varones entre 25 y 30 años muestran un aumento significativo en la violencia verbal y la coacción sexual que ejercen.

Conclusión: Las normas culturales y tradicionales pueden afectar a las relaciones violentas de pareja. Por lo que han de ser tenidas en cuenta al educar en igualdad para elaborar nuevos constructos sociales, haciendo énfasis en las mujeres con baja educación formal por ser más vulnerables de sufrir violencia de género y en los hombres del grupo etario que más la ejerce.

Palabras clave: violencia de pareja, violencia de género, cultura, sexismo.

\section{ABSTRACT:}

Objective: To examine intimate partner violence in relation to culture, age and educational level of those involved.

Methodology: A descriptive cross-sectional study involving a sample of 130 subjects, randomly selected through non-probabilistic sampling by quotas, in Spain and Peru (53 men and 77 women). An evaluation of violent intimate partner relationships was undertaken using a tool known as the "Traffic Light of Gender Violence".

Results: Significant association was found between countries and the items related to the control of dress and outings, punishment by indifference, jealousy, overprotection and control by means of the 
mobile phone. On the other hand, formal education generates a decrease in gender violence suffered in women. With regard to age, males between 25 and 30 years of age show a significant increase in verbal violence and sexual coercion.

Conclusion: Cultural and traditional rules can affect intimate partner violence. Therefore, they should be considered when educating for equality in order to elaborate new social constructs, focusing particularly on women with low levels of formal education (because they are more vulnerable to gender violence) and on men of the age group that tends to use it more.

Keywords: intimate partner violence, gender violence, culture, sexism

\section{INTRODUCCIÓN}

El análisis de las relaciones violentas de pareja se hace necesario desde distintas perspectivas, ya sea mediante el estudio del subsistema de apoyo económico, la observación de los ámbitos de residencia urbana o rural, el estudio de características en materia de educación y en el caso del presente estudio la delimitación geográfica y cultural.

Solo de esta forma se puede comprender un problema que es integral y que abarca numerosas variables a tener en cuenta. El hecho de realizar un estudio de diferenciación de relaciones violentas en distintos países implica analizar la influencia del factor cultural.

Para la limitación de los sesgos de interpretación de la información por parte de los sujetos de estudio se decidió optar por dos países hispanohablantes, sin embargo, para que existiera una diferenciación cultural se decidió escoger un país de distintos continentes. En este caso Perú de Latinoamérica y España de Europa.

El contexto de la violencia de género en Perú, según el observatorio nacional de la violencia contra las mujeres y los integrantes del grupo familiar, muestra una alta prevalencia de violencia en mujeres a mano de sus compañeros sentimentales. A pesar de esto, la violencia de género ha disminuido significativamente en la actualidad $(65,4 \%)$ con respecto al año 2009 , donde fue del $76,9 \%{ }^{(1)}$. Las formas de violencia más prevalentes en Perú son la psicológica (70-60\%), seguida de la física (30\%) y de la sexual $(6-8 \%)^{(1)}$. Se ha producido una disminución en todos los tipos de violencia en la última década. Si bien, la disminución ha sido de 11,5 puntos porcentuales en la violencia verbal o psicológica y de 7,6 puntos en la física, mientras que la violencia sexual solo ha disminuido un $2,3 \%{ }^{(1)}$.

En 2018 han existido en Perú 119 feminicidios y 241 tentativas ${ }^{(2)}$, calculándose una media de 10 víctimas de feminicidio cada mes ${ }^{(3)}$. Estos altos índices también se manifiestan en el estudio de Quispe ${ }^{(4)}$. De las violencias de género detectadas en 2018 , un $49,8 \%$ representaba violencia psicológica, un $40,1 \%$ física, un $9,6 \%$ sexual, y un $0,5 \%$ económica ${ }^{(5)}$.

El Observatorio Nacional Peruano de la Violencia Contra las Mujeres y los Integrantes del Grupo Familiar (3) indica que el 2,4\% de mujeres entre 15 y 49 años que han mantenido o tienen alguna relación, han sufrido violencia sexual en los últimos 12 meses; un $10 \%$ de violencia física, y un $15 \%$ fueron agredidas por sus parejas 0 exparejas bajo los efectos del alcohol y las drogas.

La implantación de Centros de emergencias para la mujer (CEM), ha conseguido aumentar significativamente el número de avisos atendidos por violencia de género, 
que ha pasado de unos 29.844 en el año 2006, hasta 133.697 en 2018, de las cuales un $62,7 \%$ fueron víctimas de entre 18 y 59 años ${ }^{(6)}$. Sin embargo, el $70,8 \%$ de las mujeres agredidas por sus parejas no buscaron apoyo en ninguna institución (3).

Por otra parte, en España, informes estadísticos del Ministerio de Igualdad (7) manifiestan que las denuncias por violencia de género han aumentado o se han mantenido igual en los últimos años. Mientras que en el año 2009 oscilaba entre las 135.539 denuncias; en los años 2016 y 2017 se han incrementado hasta los 143.535 y 166.260 respectivamente. Así pues, en la última macroencuesta sobre violencia de género muestra que el $4,4 \%$ de las mujeres nacidas en España sufren violencia física, el $3,7 \%$ violencia sexual, violencia psicológica emocional en un $13,6 \%$, y en un $16,3 \%$ psicológica de control y violencia económica en un $6,2 \%$. Es decir, del total de tipos de violencias de género, un $10 \%$ corresponde a la violencia física, el $8,4 \%$ a la sexual, el $67,6 \%$ a la psicológica, y un $14 \%$ a la violencia económica ${ }^{(8)}$.

Otro dato destacable de dichos informes, es el número de víctimas mortales de violencia de género desde el año 2003 hasta septiembre de 2018, que asciende hasta las 959 muertes, de las cuales 47 se han producido en este último año 2018. A pesar de todo, se observa claramente una leve tendencia de descenso de víctimas mortales con el avance de los años. Mientras que entre los años 2003 y 2010 se encuentra un número mínimo de víctimas mortales de 56 y un máximo de 76 con una media de 68 muertes por año. Entre los años 2011 y 2018 se evidencia un máximo de 62 y un mínimo de 47, lo que implica una media de 53 víctimas mortales anuales ${ }^{(7)}$.

En el ámbito educativo, se puede observar en informes del instituto de la Mujer y para la Igualdad de Oportunidades (9) que en el año 2017 la población con analfabetismo en España era mayor en mujeres que en hombres. Unas 444 mil mujeres, respecto a unos 212 mil hombres. Unas cifras reseñables a pesar de la mejora con respecto al año 2000 donde el analfabetismo en España se cifraba en 766 mil mujeres y 334 mil hombres.

También hay una diferencia en el uso del tiempo por sexos ${ }^{(10)}$. Mientras que el tiempo dedicado a cuidados personales y al estudio es similar en ambos sexos. La desigualdad entre el tiempo dedicado al hogar, la familia y el tiempo libre es evidente. En tanto que los varones disponen de 5 horas y 23 minutos de tiempo libre, mientras que las mujeres 4 horas y 32 minutos. Así mismo, el tiempo destinado al hogar y la familia en hombres se sitúan en torno a 1 hora y 54 minutos, mientras que el de la mujer se encuentra en las 4 horas y 7 minutos.

Teniendo en cuenta estos datos, debemos considerar las diferencias culturales y educativas, entre otros, como posible elemento diferenciador en el reconocimiento de actitudes violentas y sexistas en las relaciones de pareja. Es por ello que el objetivo del estudio es determinar la violencia de género sufrida y ejercida en función de la cultura, edad y nivel educativo.

\section{MATERIAL Y MÉTODO}

Estudio observacional de corte transversal descriptivo en el que se inspeccionan las actitudes violentas en relaciones de pareja en base a la cultura y tradiciones, así como la educación en países como España y Perú. Se ha usado un muestreo no probabilístico por cuotas, en el que se han recolectado 130 sujetos de edades 
comprendidas entre los 18 y 36 años. De la muestra obtenida 71 sujetos fueron de captación en Perú y 59 en España. De la muestra total la agrupación por sexos fue de 53 hombres por 77 mujeres.

Puesto que se intenta observar si existe una diferenciación cultural en la percepción de las actitudes violentas en la pareja se han tomado como variables el país, la educación y la edad. Mientras que los ítems del instrumento se han categorizado según el sexo de la muestra.

El instrumento es una adaptación del cuestionario CADRI o Conflict in Adolescent Dating Relationship Inventory, denominado "semáforo de la violencia" (11), dado que alberga tres opciones de respuesta: verde, que expresa la "no" existencia de violencia de género; amarillo, que muestra que "a veces" hay o ha existido violencia de género y finalmente rojo, que manifiesta la "si" existencia de violencia de género.

Dicho instrumento se compone de 20 preguntas tipo test con tres tipos de respuesta ("no", "a veces", y "si"), aunque para el tratamiento estadístico se han unificado las variables "A VECES" y "SI" en una sola variable, codificada con el nombre "si". Los principios del test son el reconocimiento de actividades violentas en las relaciones de pareja, entre las que se encuentran: las críticas a su forma de vestir, control coercitivo, aislamiento, acoso sexual, celos, sobreprotección y ofensas.

Las preguntas a responder en el test están divididas en función del sexo, debiendo ser respondida por ella o por él y valorando, por lo tanto, la violencia sufrida o ejercida. El cuestionario indaga aspectos como críticas a su forma de vestir, control, aislamiento, acoso sexual, celos, sobreprotección y ofensas. Las preguntas son las siguientes (Tabla 1).

Tabla 1. Cuestionario El semáforo de la violencia

\begin{tabular}{|l|l|}
\hline Pregunta 1 & $\begin{array}{l}\text { ¿Critica tu forma de vestir, de arreglarte y trata de convencerte para } \\
\text { que cambies tu aspecto? }\end{array}$ \\
\cline { 2 - 3 } & $\begin{array}{l}\text { ¿Crees que le gusta ir provocando porque se pone una ropa que } \\
\text { ella ha elegido o se arregla cómo quiere? }\end{array}$ \\
\hline Pregunta 2 & ¿Te impide ir donde quieras y con quién quieras? \\
\cline { 2 - 2 } & $\begin{array}{l}\text { ¿Le impides que decida cuándo, dónde y con quién ir porque } \\
\text { piensas que si te quiere a ti no debe salir con nadie más? }\end{array}$ \\
\hline Pregunta 3 & $\begin{array}{l}\text { ¿Intenta que te alejes de tu familia o de tus amistades o las critica y } \\
\text { descalifica? }\end{array}$ \\
\cline { 2 - 2 } & $\begin{array}{l}\text { ¿Te molesta que mantenga buenas y sólidas relaciones con su } \\
\text { familia y sus amistades? }\end{array}$ \\
\hline Pregunta 4 & ¿Te hace sentir inferior, tonta o inútil, o se burla de tus creencias? \\
\cline { 2 - 2 } & ¿Te burlas de lo que piensa, de lo que hace y/o de su trabajo? \\
\hline Pregunta 5 & ¿Te insulta o se dirige a ti con nombres ofensivos? \\
\cline { 2 - 2 } ¿Le insultas o te diriges a ella con nombres ofensivos? \\
\hline \multirow{3}{*}{ Pregunta 6 } & ¿Te ignora, te castiga o muestra indiferencia con el silencio? \\
\cline { 2 - 2 } & $\begin{array}{l}\text { ¿La ignoras, te muestras indiferente o la castigas con el silencio } \\
\text { cuando quieres demostrarle tu enfado? }\end{array}$ \\
\hline & $\begin{array}{l}\text { ¿Se pone celoso y te acusa de mantener relaciones con otras } \\
\text { personas? }\end{array}$ \\
\cline { 2 - 2 } & $\begin{array}{l}\text { ¿Te pones celoso y la acusas de mantener relaciones con otras } \\
\text { personas? }\end{array}$ \\
\hline
\end{tabular}




\begin{tabular}{|l|l|}
\hline Pregunta 8 & ¿Se muestra muy sobreprotector contigo? \\
\cline { 2 - 3 } & $\begin{array}{l}\text { ¿Crees que debes protegerla siempre de todo y de todos porque } \\
\text { piensas que ella sola no va a saber o no podrá hacerlo? }\end{array}$ \\
\hline Pregunta 9 & $\begin{array}{l}\text { ¿Te llama o manda mensajes constantemente al móvil para saber } \\
\text { qué haces, dónde estás y con quién? }\end{array}$ \\
\cline { 2 - 2 } & $\begin{array}{l}\text { ¿La llamas o le mandas mensajes constantemente al móvil para } \\
\text { saber qué hace, dónde y con quién está? }\end{array}$ \\
\hline Pregunta 10 & $\begin{array}{l}\text { ¿Te obliga a mantener relaciones sexuales o muestra insistencia } \\
\text { hasta que cedes para que se calle o porque te exige una "prueba de } \\
\text { amor" y tienes miedo de perderle? }\end{array}$ \\
\cline { 2 - 2 } & $\begin{array}{l}\text { ¿Si ella no quiere mantener relaciones sexuales sigues insistiéndole } \\
\text { porque piensas que el "no" puede ser un "quizás" o le amenazas con } \\
\text { romper la relación porque no te "demuestra su amor"? }\end{array}$ \\
\hline
\end{tabular}

También se realizó un análisis de la fiabilidad del instrumento, mediante la prueba del Alfa de Cronbach aplicado a los 10 items del instrumento, dando un resultado de 0,841 en mujeres y 0,634 en hombres, lo que otorga de media, un nivel de fiabilidad fuerte.

La recogida de datos se realizó mediante Screening masivo online en correos, redes sociales y otras aplicaciones de mensajería, y también de forma física en lugares de ámbito público. El inicio de la recolección de datos tuvo lugar entre noviembre de 2018 hasta finales de Enero de 2019. Los cuestionarios fueron autoaministrados tras una explicación sobre el estudio, sus ventajas y su carácter anónimo a la hora del tratamiento estadístico, así mismo, como la total y libre voluntariedad como requisito para participar en el estudio. El estudio cuenta con el informe favorable de la Comisión de Ética de la Investigación de la Universidad de Murcia.

El análisis de datos se realizó mediante el programa estadístico SPSS versión 22, mediante el cual se realizó un análisis descriptivo de las características de la población, así como la asociación de ítems y características mediante el uso de tablas cruzadas con la prueba de chi cuadrado de Pearson.

\section{RESULTADOS}

\section{Características de la Población de estudio}

De los 130 sujetos del estudio 71 procedían de Perú y 59 de España. El análisis de la muestra en función del País de origen, permite observar a un $54,6 \%$ de los sujetos con residencia en Perú, mientras que un 45,4\% tendría residencia en España. En referencia al sexo de la población de estudio, 53 de ellos eran hombres y 77 mujeres, lo que supone el $40,8 \%$ y el $59,2 \%$ respectivamente.

En referencia a la edad, encontramos con un valor mínimo de 18 y un valor máximo de 36 con una media de 26,56 años, situando la moda en un intervalo de edad entre los 25-30 años.

Respecto al nivel educativo de los sujetos evidencia un 3,1\% que solo cursaron la educación primaria, un $23,8 \%$ que cursaron hasta la educación secundaria. Un $70 \%$ que obtuvieron una educación superior, y un 3,1\% que cursaron otro tipo de estudios.

\section{Asociación del país con las actitudes violentas ejercidas y sufridas}

Se encontró relación entre los países de origen y ser mujer en las actitudes violentas referidas en los ítems 1, 2, 6, 7, 8 y 9. Mientras que las asociaciones por países en 
hombres se observaron en los ítems 1, 7, 8, 9 y algo menos en la pregunta 6 . En todas estas asociaciones significativas de ítems por países se reflejó un importante aumento de las respuestas afirmativas en los sujetos de Perú respecto a los de España. Todo esto, queda representado en la tabla 2, y para una mejor visualización según países y violencia sufrida o ejercida en el gráfico 1.

Tabla 2. Asociación del país con actitudes violentas ejercidas y sufridas.

\begin{tabular}{|c|c|c|c|c|c|c|c|}
\hline & \multicolumn{3}{|c|}{ Mujeres } & \multicolumn{3}{|c|}{ Hombres } \\
\hline & & No & Si & $\begin{array}{l}\text { Chi }^{2} \text { de } \\
\text { Pearson }\end{array}$ & No & $\mathbf{S i}$ & $\begin{array}{l}C_{\text {Chi }}^{2} \text { de } \\
\text { Pearson }\end{array}$ \\
\hline \multirow{3}{*}{ P1 } & Perú & $50,0 \%$ & $50,0 \%$ & 12,401 & $48,5 \%$ & $51,5 \%$ & 7,067 \\
\hline & España & $87,2 \%$ & $12,8 \%$ & Sig. & $85,0 \%$ & $15,0 \%$ & Sig. \\
\hline & & & & 0,000 & & & 0,008 \\
\hline \multirow[b]{3}{*}{ P2 } & Perú & $55,6 \%$ & $44,4 \%$ & 11,189 & $90,9 \%$ & $9,1 \%$ & 1,927 \\
\hline & & & & Sig. & & & Sig. \\
\hline & España & $89,7 \%$ & $10,3 \%$ & 0,001 & $100,0 \%$ & $0,0 \%$ & 0,165 \\
\hline \multirow[b]{3}{*}{ P6 } & Perú & $54,1 \%$ & $45,9 \%$ & 8,403 & $34,4 \%$ & $65,6 \%$ & 3,276 \\
\hline & & & & Sig. & & & Sig. \\
\hline & España & $84,6 \%$ & $15,4 \%$ & 0,004 & $60,0 \%$ & $40,0 \%$ & 0,070 \\
\hline \multirow[b]{3}{*}{ P7 } & Perú & $50,0 \%$ & $50,0 \%$ & 8,841 & $45,5 \%$ & $54,5 \%$ & 13,292 \\
\hline & & & & Sig. & & & Sig. \\
\hline & España & $82,1 \%$ & $17,9 \%$ & 0,003 & $95,0 \%$ & $5,0 \%$ & 0,000 \\
\hline \multirow[b]{3}{*}{ P8 } & Perú & $34,3 \%$ & $65,7 \%$ & 15,482 & $48,5 \%$ & $51,5 \%$ & 5,170 \\
\hline & & & & Sig. & & & Sig. \\
\hline & España & $79,5 \%$ & $20,5 \%$ & 0,000 & $80,0 \%$ & $20,0 \%$ & 0,023 \\
\hline \multirow[b]{3}{*}{ P9 } & Perú & $32,4 \%$ & $67,6 \%$ & 23,819 & $39,4 \%$ & $60,6 \%$ & 16,095 \\
\hline & & & & Sig. & & & Sig. \\
\hline & España & $87,2 \%$ & $12,8 \%$ & 0,000 & $95,0 \%$ & $5,0 \%$ & 0,000 \\
\hline
\end{tabular}


Gráfico 1. Asociación del país con actitudes violentas sufridas y ejercidas

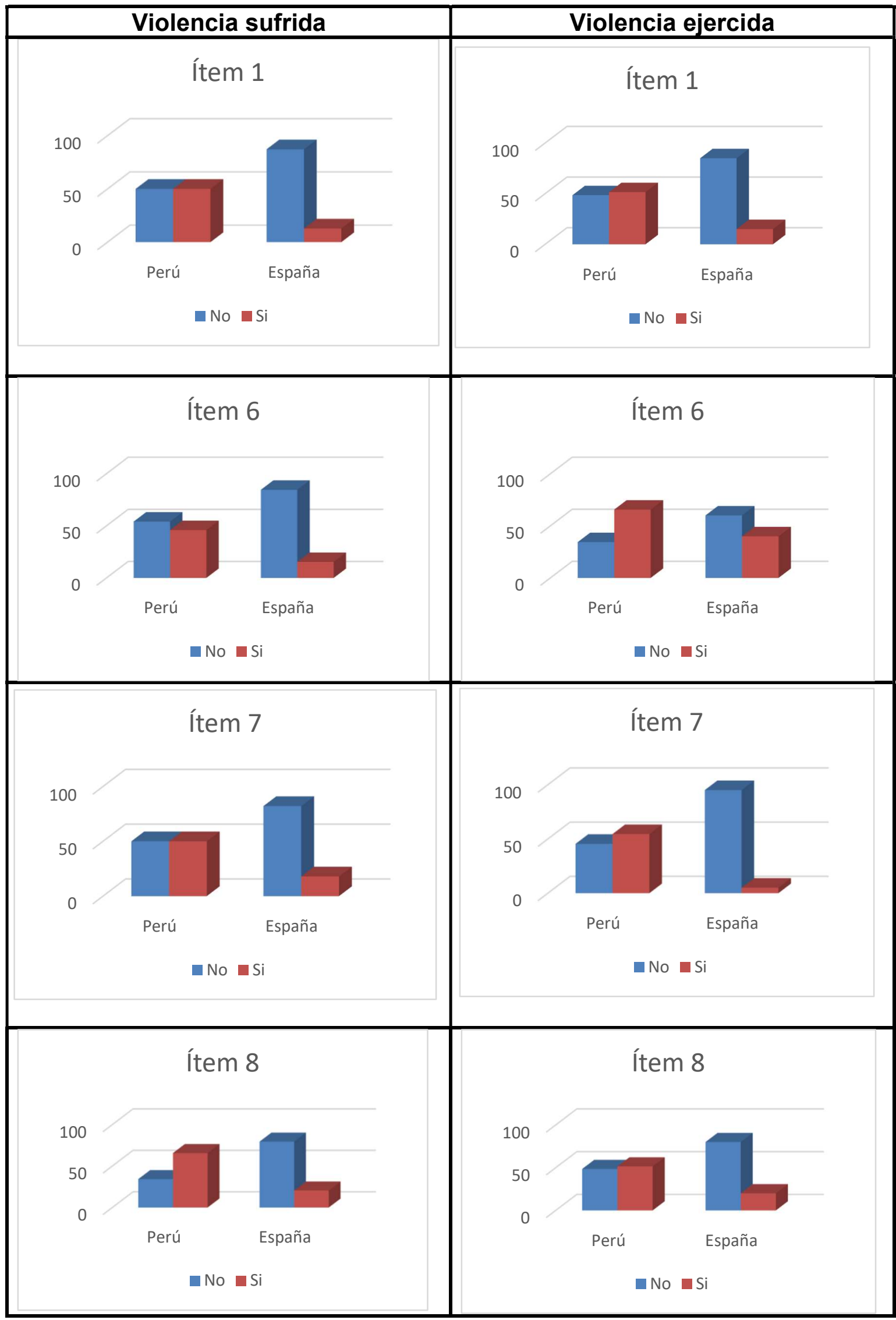




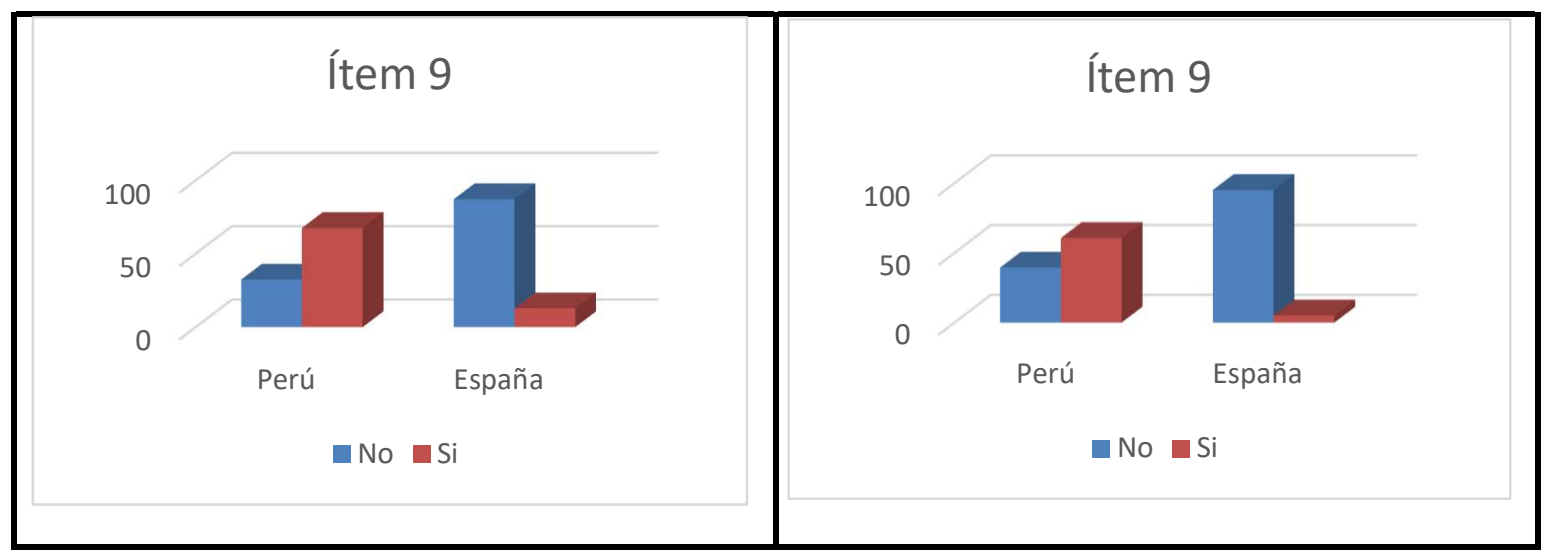

Así, se puede observar cómo en España existe una mayor disposición de respuestas negativas tanto por violencia sufrida como ejercida si tomamos en comparación con las respuestas de la muestra de Perú, donde las respuestas afirmativas tanto en violencia sufrida como ejercida superan comúnmente en todos los ítems a las respuestas negativas.

\section{Asociación educación-violencia sufrida o ejercida}

Al analizar la asociación entre los ítems del instrumento y el nivel educativo de la población de estudio, como se muestra en la tabla 3, se obtuvieron factores de asociación significativos en los ítems 1, 2, 5, 6, 7 y 9 en la muestra de mujeres. Mientras que en los hombres no se muestra asociación significativa entre los ítems y la educación formal recibida, aunque deja algunos datos reseñables en P1, P8 y P9. Esto, implica que a mayor nivel de educación formal existe menor violencia de género contra las mujeres, mientras que en el caso de los hombres la educación formal no implica una disminución de la violencia de género ejercida.

Tabla 3. Asociación educación-violencia sufrida o ejercida

\begin{tabular}{|c|c|c|c|c|c|c|c|}
\hline & \multirow{3}{*}{\begin{tabular}{|l|} 
Otros \\
$1,9 \%$ \\
\end{tabular}} & \multirow{3}{*}{$\begin{array}{c}\text { E. } \\
\text { Primaria } \\
0,0 \%\end{array}$} & \multirow{3}{*}{$\begin{array}{c}\text { E. } \\
\text { Secundaria } \\
7,5 \%\end{array}$} & \multirow{3}{*}{$\begin{array}{c}\text { E. } \\
\text { Superior } \\
90,6 \%\end{array}$} & \multirow{3}{*}{$\begin{array}{c}\text { Chi }^{2} \\
27,17 \\
2\end{array}$} \\
\hline & & & & & & & \\
\hline \multirow{6}{*}{ P1 } & \multirow[t]{3}{*}{ mujeres } & No & & & & & \\
\hline & & $\mathrm{Si}$ & $0,0 \%$ & $4,2 \%$ & $58,3 \%$ & $37,5 \%$ & Sig. \\
\hline & & & & & & & 0,000 \\
\hline & \multirow{3}{*}{ hombres } & No & $9,1 \%$ & $0,0 \%$ & $24,2 \%$ & $66,7 \%$ & 6,857 \\
\hline & & \multirow[t]{2}{*}{$\mathrm{Si}$} & \multirow[t]{2}{*}{$0,0 \%$} & \multirow[t]{2}{*}{$15,0 \%$} & \multirow[t]{2}{*}{$25,0 \%$} & \multirow[t]{2}{*}{$60,0 \%$} & Sig. \\
\hline & & & & & & & 0,077 \\
\hline \multirow{6}{*}{ P2 } & \multirow{3}{*}{ mujeres } & \multirow[t]{2}{*}{ No } & \multirow[t]{2}{*}{$1,8 \%$} & \multirow[t]{2}{*}{$0,0 \%$} & \multirow[t]{2}{*}{$16,4 \%$} & \multirow[t]{2}{*}{$81,8 \%$} & 8,141 \\
\hline & & & & & & & Sig. \\
\hline & & $\mathrm{Si}$ & $0,0 \%$ & $5,0 \%$ & $40,0 \%$ & $55,0 \%$ & 0,043 \\
\hline & \multirow{3}{*}{ hombres } & No & $6,0 \%$ & $4,0 \%$ & $24,0 \%$ & $66,0 \%$ & 5,054 \\
\hline & & \multirow[b]{2}{*}{$\mathrm{Si}$} & \multirow[b]{2}{*}{$0,0 \%$} & \multirow[b]{2}{*}{$33,3 \%$} & \multirow[b]{2}{*}{$33,3 \%$} & \multirow[b]{2}{*}{$33,3 \%$} & Sig. \\
\hline & & & & & & & 0,168 \\
\hline & \multirow[t]{2}{*}{ mujeres } & \multirow[t]{2}{*}{ No } & \multirow[t]{2}{*}{$1,4 \%$} & \multirow[t]{2}{*}{$0,0 \%$} & \multirow[t]{2}{*}{$22,9 \%$} & \multirow[t]{2}{*}{$75,7 \%$} & $\begin{array}{c}12,50 \\
4\end{array}$ \\
\hline & & & & & & & Sig. \\
\hline
\end{tabular}




\begin{tabular}{|c|c|c|c|c|c|c|c|}
\hline \multirow[t]{3}{*}{ P5 } & & $\mathrm{Si}$ & $0,0 \%$ & $16,7 \%$ & $33,3 \%$ & $50,0 \%$ & 0,006 \\
\hline & \multirow[b]{2}{*}{ hombres } & No & $6,3 \%$ & $4,2 \%$ & $25,0 \%$ & $64,6 \%$ & 2,379 \\
\hline & & $\mathrm{Si}$ & $0,0 \%$ & $20,0 \%$ & $20,0 \%$ & $60,0 \%$ & Sig. \\
\hline \multirow{6}{*}{ P6 } & \multirow{3}{*}{ mujeres } & No & $1,9 \%$ & $0,0 \%$ & $15,1 \%$ & $83,0 \%$ & 8,383 \\
\hline & & & & & & & Sig. \\
\hline & & Si & $0,0 \%$ & $4,3 \%$ & $39,1 \%$ & $56,5 \%$ & 0,039 \\
\hline & \multirow{3}{*}{ hombres } & No & $8,7 \%$ & $0,0 \%$ & $21,7 \%$ & $69,6 \%$ & 3,134 \\
\hline & & & & & & & Sig. \\
\hline & & $\mathrm{Si}$ & $3,4 \%$ & $10,3 \%$ & $24,1 \%$ & $62,1 \%$ & 0,371 \\
\hline \multirow{6}{*}{ P7 } & \multirow{3}{*}{ mujeres } & No & $2,0 \%$ & $0,0 \%$ & $11,8 \%$ & $86,3 \%$ & 14,24 \\
\hline & & & & & & & $\begin{array}{c}4 \\
\operatorname{Sig}\end{array}$ \\
\hline & & $\mathrm{Si}$ & $0,0 \%$ & $3,8 \%$ & $46,2 \%$ & $50,0 \%$ & 0,003 \\
\hline & \multirow{3}{*}{ hombres } & No & $8,8 \%$ & $2,9 \%$ & $23,5 \%$ & $64,7 \%$ & 2,959 \\
\hline & & & & & & & Sig. \\
\hline & & $\mathrm{Si}$ & $0,0 \%$ & $10,5 \%$ & $26,3 \%$ & $63,2 \%$ & 0,398 \\
\hline \multirow{6}{*}{ P9 } & \multirow{3}{*}{ mujeres } & No & $0,0 \%$ & $0,0 \%$ & $10,9 \%$ & $89,1 \%$ & 14,92 \\
\hline & & & & & & & $\frac{0}{c_{i}}$ \\
\hline & & $\mathrm{Si}$ & $33 \%$ & $3.3 \%$ & $43.3 \%$ & $50.0 \%$ & 0.002 \\
\hline & \multirow{3}{*}{ hombres } & No & $9.4 \%$ & $0.0 \%$ & $21.9 \%$ & $68.8 \%$ & 7.038 \\
\hline & & & & & & & Sig. \\
\hline & & Si & $0,0 \%$ & $14,3 \%$ & $28,6 \%$ & $57,1 \%$ & 0,071 \\
\hline
\end{tabular}

\section{Asociación intervalos de edad-violencia sufrida o ejercida}

Finalmente, en el análisis para la asociación de ítems con los intervalos de edad de las muestras según los sexos, se ha podido observar una nula asociación entre los intervalos de edad y las respuestas ofrecidas por las mujeres. Sin embargo, en los hombres se aprecia una asociación en P5 y P10 a la respuesta "si" con una significancia en la fuerza de asociación de 0,015 y 0,037 respectivamente en los intervalos de edad de 25 a 30 años. De esta forma se ha podido observar que el $24 \%$ y un 19\% respectivamente de los hombres entre 25 y 30 años respondieron afirmativamente los ítems relacionados con insultos a la pareja y coacción sexual, mientras que en el resto de franjas de edad respondieron de forma negativa. Otro dato que muestra una relativa significancia es el correspondiente al ítem 7 manifestado por los celos y que se muestra con mayor índice en el grupo de hombres con un intervalo de edad de entre 18 y 24 años, llegando a manifestar este tipo de violencia en un $52 \%$ de los hombres que pertenecían a esta franja de edad.

\section{DISCUSIÓN}

Entre los resultados de este estudio se puede evidenciar por lo tanto que existe una diferenciación de los índices de casos de violencia de género en función de la cultura del país de muestreo. Así, se han observado tasas más altas de violencia en todos los ítems en la muestra de Perú que en la de España, lo que concuerda con la bibliografía ${ }^{(8)}$ y datos estadísticos expuestos en la introducción del estudio. Estas 
asociaciones significativas se hacen más fuertes en los ítems relacionados con el control de la vestimenta y de las salidas, el castigo mediante indiferencia, los celos, la sobreprotección y el control a través del móvil.

El elevado índice de estos ítems en los sujetos de Perú, también queda reflejado en el estudio de Blitchtein-Winicki(12), donde se identifican como los principales factores asociados a la violencia de género las normas tradicionales como el control por el hombre de las visitas a familiares y amigos por parte de la mujer, el sentirse amenazado por relaciones previas de la pareja, el hecho de que la mujer tenga mayor educación formal que él o la mayor toma de decisiones en el hogar.

Aunque esta problemática es evidenciada en ambos países, se acentúa aún más en el país latinoamericano, donde las víctimas de violencia de género están más desprotegidas debido a que su legislación solo reconoce la violencia en la medida en que se ocasionan daños y lesiones mediante resultados observables ${ }^{(13)}$.

Por otra parte, un estudio en países de la UE observa que la violencia doméstica y la desigualdad de género en la política no poseen un diagnóstico adecuado puesto que se ofrecen diversas soluciones pero no se explican realmente los agentes causantes de estos $^{(14)}$.

Respecto a la asociación de los diversos ítems con el nivel de educación de los sujetos de la muestra se observa como las mujeres de este estudio que poseen una mayor formación sufrían un menor nivel de violencia de género. Esto coincide con el estudio de González Sala ${ }^{(15)}$, donde se evidencia que los grupos con una educación formal de mayor nivel sufren menores niveles de violencia de género y tienen menor tolerancia hacia este tipo de actitudes. Además, el hecho de que las mujeres posean un mayor nivel de educación formal desempeña un papel importante en las condiciones laborales a las que pueden acceder. Sin embargo, este hecho, lejos de mejorar las condiciones de conciliación en las labores del hogar, lo que ha producido es una devaluación e invisibilidad de las tareas domésticas que finalmente acaban siendo relegadas a asistentas en su mayoría mujeres de sectores más pobres y con menor educación, perpetuando así el problema y favoreciendo así el rechazo masculina a participar en dichas labores ${ }^{(16)}$.

En el análisis por intervalos de edad se distinguen ciertos patrones de conducta violenta entre los sujetos varones de entre 25 y 30 años, en los que se manifiesta una mayor tendencia a insultos e imposiciones sexuales hacia sus parejas. Esto implica a un $24 \%$ y un $19 \%$ de los varones situados en el citado intervalo de edad, lo cual es una gran problemática para afrontar en adultos jóvenes. Así pues, esto se relaciona con una serie de estudios(17-19), que manifiestan que entre el 25 y el $27 \%$ de las mujeres violentadas sexualmente se encontraban en los rangos de edad de entre 20 y 35 años. También, un estudio similar en adolescentes Españolas ofreció porcentajes equivalentes en mujeres víctimas de violencia sexual entre los 15 y 19 años ${ }^{(20)}$, y otro estudio en el contexto peruano muestra que un $43,1 \%$ de las adolescentes podrían estar obligadas a tener sexo forzado(21).

Este tipo de acciones suelen ser predictores de la violencia física, sin embargo, López Zafra $^{(22)}$ muestra que las edades más proclives para este tipo de violencia se encuentran entre los sujetos más mayores, con franjas de edad de entre 51 años en adelante y en los más jóvenes de edades inferiores a los 21 años. Así mismo, un estudio en parejas adolescentes del norte de España(23) muestra mayor violencia 
sexual entre las chicas jóvenes con relaciones de pareja violentas, donde hasta un $60 \%$ de ellas afirmó haber sufrido casos de coacción sexual. Y otro estudio(24), expresa que los hombres manifiestan conductas más agresivas a mayor tiempo de relación. Es por ello que se podrían establecer posibles casos de violencia física futura en los sujetos de nuestra muestra, aunque pueden variar según la cultura de estudio.

Por lo tanto, la cultura entendida en el sentido más amplio de la palabra como tradición son conocimientos, creencias y costumbres repetidos a lo largo del tiempo e incluidos en el estrato más profundo de la identidad social colectiva. Esto genera hábitos y tendencias que pautan la forma de pensar y actuar en la vida cotidiana del individuo.

Johan Galtung en su obra(25), nos habla de dos tipos de violencia. Por un lado, la violencia visible o directa, en la que es evidente el acto y los agentes en los que se manifiesta la agresión. Por otro lado, tenemos lo que Galtung califica como violencia invisible, teniendo éste como base los constructos culturales que se manifiestan mediante el menor respeto por la naturaleza no humana, efectos espirituales, daños a la estructura social y mundial, transferencia de estructuras a la siguiente generación, y cultura violenta del trauma y la gloria.

De esta forma el autor incide en que la violencia directa refuerza la violencia estructural y cultural.

Por otra parte Glynis Breackwell ${ }^{(26)}$ explica cómo los actos violentos son legitimados por los estereotipos culturales de género y por su prevalencia en el tiempo. La implantación de los actos violentos de género en el sustrato estructural de la sociedad como explica Gatlung y su legitimización por presencia cultural a lo largo del tiempo como indica Breackwell, hace de éste un problema realmente difícil de erradicar.

Además, estos constructos culturales invisibilizan aún más la violencia de género transformando la violencia visible que explicaba Gatlung en algo que debía llevarse en la intimidad del núcleo familiar. Así, Rafaela Vos Obeso ${ }^{(27)}$ expone como la violencia de género debía ser resuelta según las "costumbres" en la zona intima del hogar.

Respecto a cómo atajar este problema, Espinar Ruiz ${ }^{(28)}$ da en la clave al tratar el tema de forma que explica que esta construcción cultural que permite la violencia en la pareja, se debe en gran medida a los procesos de socialización diferencial, donde se transmiten y aprenden mensajes diferentes en función del sexo del destinatario.

De esta forma nos encontramos ante un aprendizaje social e individual de comportamientos, roles e identidades adjudicados en función del sexo y que finalmente se traduce en desigualdades en las relaciones de género.

Con respecto a las culturas que alberga este estudio, Rivers y Peristiany ${ }^{(29)}$ dicen:

$<<$ los patrones culturales de las sociedades Mediterráneas y de América del Sur han sido denominadas culturas del Honor, por la gran importancia que dan a las estructuras sociales tradicionales y el peso que tiene la opinión pública como estamento social. En estas culturas los hombres son los encargados de cuidar de la familia, y proteger a las mujeres de conductas deshonrosas, normalmente asociadas con conductas sexuales. 
La defensa de las mujeres de la familia llega a anularlas o incluso a humillarlas tratándolas como una propiedad más de la familia. El uso de la violencia es normal, en forma de amenaza o de agresión si no se cumplen las normas que preservan la reputación de la familia>>

De esta forma surge la Teoría de la cultura del honor donde los ideales culturales y emocionales surgen como legitimadores de la violencia de pareja, que son aprendidos de forma colectiva, pero lo cual también puede usarse para prevenir este tipo de acciones $^{(30)}$.

\section{CONCLUSIONES}

Los resultados obtenidos permiten ver una elevada asociación entre cultura y la violencia sufrida y ejercida, especialmente en los ítems relacionados con control de la vestimenta y de las salidas, el castigo mediante indiferencia, los celos, la sobreprotección y el control a través del móvil. En el caso de la muestra de Perú se han mostrado mucho más elevados estos ítems que en la muestra de España lo que puede implicar un aprendizaje social distinto basado en normas culturales y tradicionales pueden afectar a las relaciones de género.

Respecto al nivel de educación formal se ha podido observar como ésta se manifiesta como un factor protector de la violencia de género en las mujeres del estudio, disminuyendo el número de respuestas afirmativas en los ítems relacionados con el control de la vestimenta y de las salidas, insultos, castigo mediante indiferencia, celos, y control a través del móvil tanto en la población española como peruana. Sin embargo, en los hombres no se observa una asociación significativa, ya sea en el aumento o en la disminución de los ítems. Lo que induce a pensar que los mecanismos que van asociados a la violencia de género, van más allá de lo que implica el nivel de educación formal.

En los varones con edades comprendidas entre los 25 y 30 años se han observado ciertas características violentas como son los insultos e imposiciones sexuales a sus parejas, lo cual, no se ha encontrado en otros rangos de edad.

Entre las conclusiones que se deben extrapolar responden claramente a la actuación sobre los constructos sociales que determinan los roles de género, dado que estos conocimientos colectivos y aunque de difícil erradicación pueden combatirse con nuevos constructos sociales que si son prevalentes en el tiempo puede erradicar la Cultura del Honor.

\section{REFERENCIAS}

1. Familiar ONdIVcIMylldG. Indicators of the demographic and family health survey (ENDES) Lima, Peru: Ministry of Women and Vulnerable Populations; 2017. Available from: https://observatorioviolencia.pe/datos-inei-2017-2/.

2. Vulnerables MdIMyP. Violence in figures: Statistical report of October 2018 Lima2018.

Available from: https://www.mimp.gob.pe/files/programas nacionales/pncvfs/publ icaciones/informe-estadistico-07-2018 PNCVFS-UGIGC.pdf.

3. Familiar ONdIVcIMylldG. Data and evidence on violence against women and to the members of the family group according to information source Lima, Peru: 
Ministry of Women and Vulnerable Populations; 2017. Available from: https://observatorioviolencia.pe/datos/.

4. Ilanzo Q, Pamela M, Curro Urbano OM, Cordova Delgado M, Pastor Ramirez N, Puza Mendoza GM, et al. Extreme violence against women and femicide in Peru. Cuban Journal of Public Health. 2018; 44: 278-294.

5. Vulnerables MdIMyP. National Summary: National Program against family and sexual violence. Period Jan-Dec 2018 Lima2018. Available from: https://portalestadistico.pe/por-regiones-2018/.

6. Family Ondlvclmylidg. Administrative records of the national program against family and sexual violence of the mimp Lima, Peru: Ministry of women and vulnerable $\quad 2017 . \quad$ Available from: https://observatorioviolencia.pe/datos-2/datospncvfs/.

7. Government delegation for gender violence of the Ministry of Sanitation.

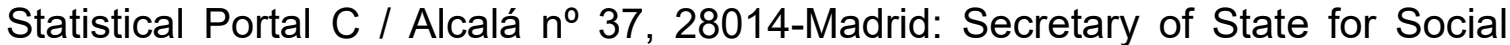
Services and Equality; $2018 . \quad$ Available from: http://estadisticasviolenciagenero.igualdad.mpr.gob.es/.

8. Mdssse equality. Macro survey of violence against women 2015. Madrid2015. p. 248

9. Idlmyplid opportunities. Women in figures - Education C / Condesa de Venadito, 34. 28027 Madrid .: Secretary of State for Equality; 2018. Available from: http://www.inmujer.gob.es/MujerCifras/Educacion/NivelEstudios.htm.

10. Idlmyplid opportunities. Women in figures - Conciliation - Uses of time C / Condesa de Venadito, 34. 28027 Madrid .: State Secretariat for Equality; 2018. Available from: http://www.inmujer.gob.es/MujerCifras/Conciliacion/UsosdelTiempo.htm.

11. Old CM, Mas AM, Cerdá ME, Sánchez NM, Corbí RG, editors. Exploration of violence in young university couple relationships 2012: Institute of Education Sciences.

12. Blitchtein-Winicki D, Reyes-Solari E. Factors associated with recent physical violence of a couple towards women in Peru, 2004-2007. Peruvian Journal of Experimental Medicine and Public Health. 2012; 29 (1): 35-43.

13. Sagot M. Critical Path of Women Affected by Domestic Violence in Latin America: Case Studies from Ten Countries: Pan American Health Org; 2000

14. Bustelo $M$, Lombardo $E$. The 'interpretative frameworks' of equality policies in Europe: conciliation, violence and gender inequality in politics. Spanish Journal of Political Science. 2006 (14): 117-140.

15. Sala-Doi FG, Valero BM. Perception of gender violence according to educational level and gender. Psychological Information. 2014 (108): 68-81.

16. Fuller N. Changes and Permanences in Gender Relations in Peru. Family and private life Transformations, tensions, resistances and new senses. 2005: 107129.

17. Rivera Lavalle CJ. Sociodemographic characteristics types and attitudes towards violence in women of fertile age, health post Chalacala Alta-Bellavista-Sullana 2018. 2018.

18. Zapata S, Eveling L. Sociodemographic characteristics, types and attitude toward violence in women of childbearing age, from the Marcavelica-Sullana health centre 2018. 2018.

19. Arismendiz P, Danitza B. Types sociodemographic characteristics and attitude towards violence in women of childbearing age of the health centre of Somate Bajo-Bellavista 2018. 2018. 
20. Garcia C, Juvitza L. Sociodemographic characteristics types and attitude towards violence in adolescent women of the health center 9 de Octubre-Sullana 2018. 2018.

21. Vargas F, Sivoyny J. Sexual knowledge and attitudes in adolescents of the human settlement October 9 Sullana-Piura, 2018.

22. López-Zafra E. Relationship between Culture of Honor and Gender Identity: the role of sex, age and educational level in predisposition to violence. Psychology studies. 2008; 29 (2): 209-220.

23. Rodríguez-Castro $\mathrm{Y}$, Lameiras-Fernández $\mathrm{M}$, Carrera-Fernández MV, AlonsoRuido P. Violence in the relationships of Galician adolescents. Journal of Studies and Research in Psychology and Education. 2017 (02): 010-13.

24. Roche FP, Llor AMS, Bravo MdMP, Meseguer CB. Identification of adolescent violence as a control mechanism in the classroom and in courtship. NURE research: Nursing Scientific Magazine. 2016; 13 (82): 4.

25. Galtung J. Violence, war and its impact. On the visible and invisible effects of violence. 2004; 5 .

26. Breakwell GM. Coping with aggressive behaviour: Universities Press; 1997

27. Obeso RV. Traditions And Cultures: Accomplices Of Violence Against Women. Amauta. 2013; 10 (19).

28. Espinar-Ruiz E. The sociocultural roots of gender violence. 2007

29. Pitt-Rivers J, Peristiany JG. Honor and Grace: Editorial Alliance; 1993

30. López-Zafra E. Culture of Honor: Influence of sociodemographic variables and Psychosocial dimensions in the predisposition to react with violence to offenses to honour. Violence against women: description Biopsychosocial intervention. 2009: 117-142. 